\title{
A NOTE ON ROC ANALYSIS AND NON-PARAMETRIC ESTIMATE OF SENSITIVITY
}

\author{
JUN ZHANG
}

\author{
UNIVERSITY OF MICHIGAN AT ANN ARBOR
}

SHANE T. MUELLER

UNIVERSITY OF MICHIGAN AT ANN ARBOR ${ }^{\dagger}$

\begin{abstract}
In the signal detection paradigm, the non-parametric index of sensitivity $A^{\prime}$, as first introduced by Pollack and Norman (1964), is a popular alternative to the more traditional $d^{\prime}$ measure of sensitivity. Smith (1995) clarified a confusion about the interpretation of $A^{\prime}$ in relation to the area beneath proper receiver operating characteristic (ROC) curves, and provided a formula (which he called $A^{\prime \prime}$ ) for this commonly held interpretation. However, he made an error in his calculations. Here, we rectify this error by providing the correct formula (which we call $A$ ) and compare the discrepancy that would have resulted. The corresponding measure for bias $b$ is also provided. Since all such calculations apply to "proper" ROC curves with non-decreasing slopes, we also prove, as a separate result, the slope-monotonicity of ROC curves generated by the likelihood-ratio criterion.
\end{abstract}

Key words: signal detection theory, $A^{\prime}$, non-parametric estimate of sensitivity, likelihood-ratio

\section{Introduction}

Signal detection theory (Tanner \& Swets, 1954; Peterson, Birdsall, \& Fox, 1954; Swets, Tanner, \& Birdsall, 1964; Green \& Swets, 1964) is commonly used to interpret data from tasks in which stimuli (e.g., tones, words, or medical images) are presented to an observer who must determine which one of the two categories (e.g., present or absent, old or new, benign or malignant) the stimulus belongs to. This procedure yields two measures of behavioral performance: the hit rate $(H)$ and the false alarm rate $(F)$. Normally, $H$ and $F$ are transformed into indices of sensitivity and bias based on assumptions about an underlying statistical model (e.g., the $d^{\prime}$ index under the assumption of normal distributions with equal variance). However, other estimates are available that avoid making distributional assumptions. The most prominent alternative is based on an estimate of the "average" area of possible receiver operating characteristic (ROC) curves that are constrained by the experimental data $\left(H\right.$ and $F$ ). This measure (called $A^{\prime}$ ) was first proposed by Pollack and Norman (1964) and popularized by Grier (1971).

Since its introduction, $A^{\prime}$ has become a popular "non-parametric" alternative to $d^{\prime}$ for measuring sensitivity in detection and categorization tasks (Macmillan \& Creelman, 1996). Its popularity is probably a consequence of the fact that, unlike $d^{\prime}$, it is defined even when $F$ or $H$ is equal or close to 0 or 1 . Nonetheless, given its widespread use, it is interesting to note that $A^{\prime}$ does not measure what many people believe it measures (Smith, 1995). Smith reported that $A^{\prime}$ was commonly (and incorrectly) believed to be an average between the minimum-area and maximum-area proper ROC curves that can pass through the observed point $p=(F, H)$. He then proposed a new measure, $A^{\prime \prime}$, which he claimed to be the correct formula for this common interpretation. However, Smith made an error in his calculation, so that the formula he gave does not always reflect the average of the minimum and maximum proper ROC curves that pass through a given

Requests for reprints should be sent to Jun Zhang, Department of Psychology, University of Michigan, 525 East University, Ann Arbor, MI 48109, USA. E-mail: junz@umich.edu

${ }^{\dagger}$ Now at Department of Psychology, 1101 East 10th Street, Indiana University, Bloomington, IN 47405, USA 
point.

In this note, we will provide an appropriate formula (which we call $A$ ) for this sensitivity measure, and compare the discrepancy between $A^{\prime}, A^{\prime \prime}$, and $A$. We will also introduce an associated bias estimate (which we call $b$ ). To investigate the slope-monotonicity assumption (the definition of a "proper" ROC curve), we will first show that ROC curves based on the likelihoodratio criterion, despite a possibly non-monotonic relation between the sensory encoding values and the likelihood-ratio values, always have monotonic slope. We then apply the Neyman-Pearson Lemma, which implies that likelihood-ratio based ROC curves always lie on or above ROC curves based on other criteria, to argue that $A$ represents an optimistic estimate of detection sensitivity from subjects.

\section{Estimates of Sensitivity Based on ROC Areas}

One way to obtain an estimate of sensitivity is to measure the area under the ROC curve (Green \& Swets, 1964). To obtain this area, response bias is systematically manipulated (e.g., by varying the payoff) and $(F, H)$ pairs are plotted for each bias condition. Then, an ROC curve is formed from these points, either by fitting a curve through the observed points based on an underlying model, or by simply connecting the observed data points. The area under the obtained curve is taken as an index of sensitivity which is justified, because this area has been shown to be equal to the percentage correctly obtained in a two-alternative forced-choice task (e.g., Green \& Swets, 1964, p. 47). As with any statistic, ROC areas may vary due to sampling error. This type of variability was investigated by Pollack and Hsieh (1969) for ROC curves based on multiple data points. Using ROC areas to estimate sensitivity is less tractable if data are only obtained for a single bias condition, because there is a large range of "proper" ROC curves ${ }^{1}$ that could pass through any single point. However, for the point $p=(F, H)$, as seen in Figure 1, all proper ROC curves must fall within or on the bounds of light shaded regions $A_{1}$ and $A_{2}$. Pollock and Norman (1964) proposed a measure of sensitivity $A^{\prime}$ as the average of the areas $A_{1}+I$ and $A_{2}+I$, which Grier (1971) explicitly showed to equal:

$$
A^{\prime}=\frac{1}{2}+\frac{(H-F)(1+H-F)}{4 H(1-F)} .
$$

Generalization of the $A^{\prime}$ measure to $A_{r}^{\prime}$, namely the averaged area under ROC curves passing through multiple data points (obtained by the confidence rating paradigm), was considered by Donaldson and Good (1996). It is noteworthy that the area $A_{1}+I$ and $A_{2}+I$ (used in the construction of $\left.A^{\prime}\right)$ is, respectively, a function of odds ratios $F / H$ and $(1-H) /(1-F)$ which in turn are interpretable with respect to the logistic model (Macmillan \& Creelman, 1996).

However, $A^{\prime}$ (and $A_{r}^{\prime}$ ) is not the average of the maximum-area and minimum-area proper ROC curves, although it is easily shown to be the average of the area $I$ and $I+A_{1}+A_{2}$ from Figure 1. While the smallest area associated with a proper ROC curve passing through $p$ is $I$, the area of the largest proper ROC curve, namely $A_{\max }$, is not $I+A_{1}+A_{2}$, and its proper calculation is a much subtler issue. Smith (1995) made the claim (without proof) that, depending on whether $p$ is to the left or right of the negative diagonal $H+F=1, A_{\max }$ is the larger of $I+A_{1}$ and $I+A_{2}$, shown in Figure 1. Smith (1995) then derived the average of $I$ and $\max \left\{I+A_{1}, I+A_{2}\right\}$, which he called $A^{\prime \prime}$, as

\footnotetext{
${ }^{1}$ A proper (or admissible) ROC curve is a concave curve that connects the end points $(0,0)$ and $(1,1)$. It necessarily lies above the positive diagonal $H=F$, and has non-increasing slope going from $(0,0)$ to $(1,1)$.
} 


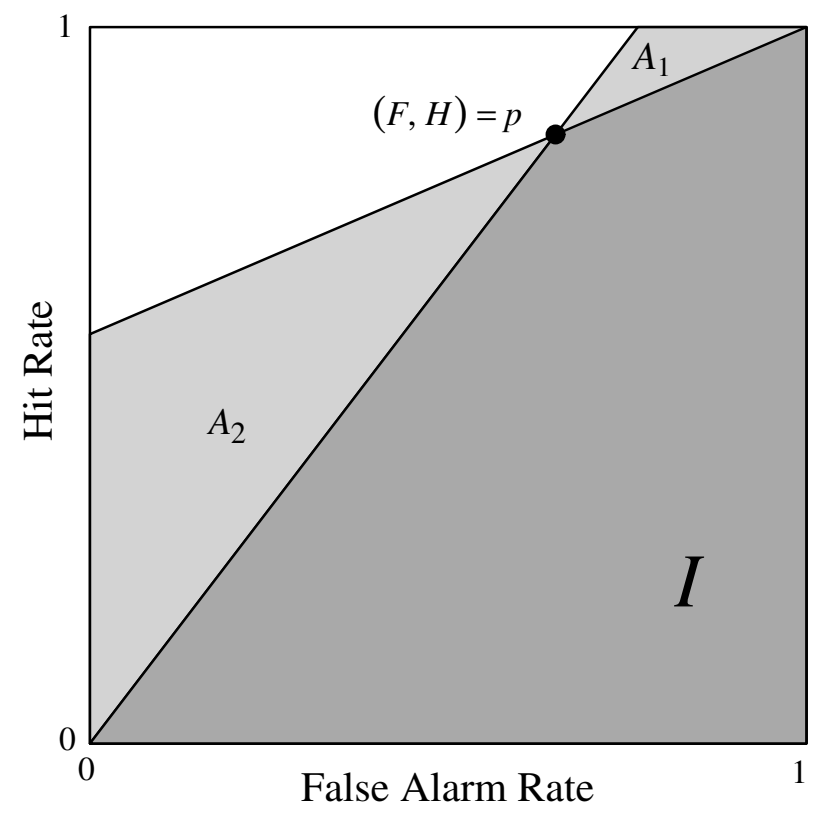

FIGURE 1.

Proper ROC curves through point $p$ must lie within or on the boundaries of the light shaded regions $A_{1}$ and $A_{2}$. The minimum-area proper ROC curve through $p$ lies on the boundary of region $I$.

$$
A^{\prime \prime}= \begin{cases}\frac{3}{4}+\frac{H-F}{4}-\frac{F}{4 H} & \text { if } \quad H \leq(1-F) \\ \frac{3}{4}+\frac{H-F}{4}-\frac{1-H}{4(1-F)} & \text { if } \quad H>(1-F),\end{cases}
$$

However, Smith's (1995) claim about $A_{\max }$ was erroneous when $p$ is in the upper left quadrant of the ROC space (i.e., $F \leq 0.5$ and $H \geq 0.5$ ). In this region, neither $I+A_{1}$ nor $I+A_{2}$ is the region bounded by the maximum-area proper ROC curve passing through $p$. In the next section, we will derive the correct formula for the maximum-area ROC curve for $p$ in any region, and introduce a new formula (denoted as $A$ ) for the average of the maximum and minimum area of proper ROC curves passing through a given data point.

The Proper ROC Curve with Maximum Area

Proposition 1. The maximum area $A_{\max }$ among all proper ROC curves passing through a given point $(F, H)$ is

$$
A_{\max }=\left\{\begin{array}{lll}
1-2 F(1-H) & \text { if } & F \leq 0.5 \leq H, \\
1-\frac{F}{2 H} & \text { if } & F \leq H<0.5, \\
1-\frac{1-H}{2(1-F)} & \text { if } & 0.5<F \leq H .
\end{array}\right.
$$

Proof. To determine the value for $A_{\max }$ achieved by proper (admissible) ROC curves passing through $p=(F, H)$, we examine the value of its slope at $p$, denoted $s_{p}$. Because the slope of a proper ROC curve is non-increasing as one progresses from $(0,0)$ to $(1,1)$, its slope at $p$ must satisfy 


$$
\frac{H}{F} \leq s_{p} \leq \frac{1-H}{1-F} .
$$

The upper bound of the slope occurs when the line passes through $(0,0)$ and $p=(F, H)$, and the lower bound of the slope occurs when the line passes through $(1,1)$ and $p=(F, H)$.

In the case that the slope at $p$ is undefined (i.e., an ROC curve is continuous but not smooth at $p$, which is possible), then its left and right slopes (by taking the tangent from the left and right sides) exist and their values $s_{p}^{\mathrm{L}}, s_{p}^{\mathrm{R}}$ are constrained by the above inequality as well, with $s_{p}^{\mathrm{L}}>s_{p}^{\mathrm{R}}$.

We now classify all proper ROC curves passing through $p$ according to the value of $s_{p}$ when the slope is undefined, then such a curve may be put into an arbitrary class whose $s_{p}$ value satisfies $s_{p}^{\mathrm{L}} \geq s_{p} \geq s_{p}^{R}$. For each $s_{p}$, we then construct the piecewise linear ROC curve $\mathcal{C}_{p}$ which consists of (in the most general case) three straight segments, like the one in Figure 2, with the middle segment having a slope of $s_{p}$. When $s_{p}=(1-H) /(1-F)$ or $s_{p}=H / F$, then either the first or the third segment degenerates to a point, respectively, so that the piecewise linear function has only two straight segments (as seen in Figure 1). It is obvious that the area beneath such a piecewise linear ROC curve, for a given $s_{p}$ value, would be larger than for any other proper ROC curve in the corresponding class (i.e., with the same $s_{p}$ ).

To calculate the area beneath such an ROC curve, i.e., the area of the polygon, we denote the intersection of this middle segment with the boundary line $H=1$ and $F=0$ at $(x, 1)$ and $(0,1-y)$, respectively, where

$$
\frac{F}{x}=\frac{t_{1}}{t_{1}+t_{2}}=\tau, \quad \frac{1-H}{y}=\frac{t_{2}}{t_{1}+t_{2}}=1-\tau
$$

with

$$
F \leq \tau \leq H
$$

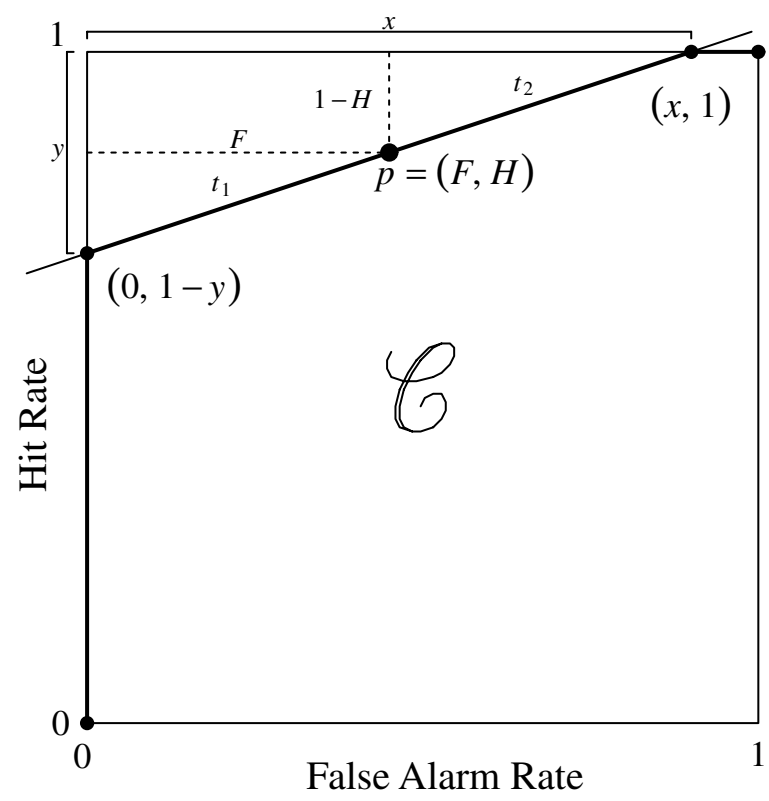

FIGURE 2.

Example of a proper ROC curve through $p$. The ROC curve $\mathcal{C}$, a piecewise linear curve denoted by the dark outline, is formed by following a path from $(0,0)$ to $(0,1-y)$ to $(x, 1)$ and on to $(1,1)$. 
(because $x \leq 1$ and $y \leq 1$ ). Now the ROC area is

$$
A(\tau)=1-\frac{1}{2} x y=1-\frac{F(1-H)}{2} \frac{1}{\tau(1-\tau)}
$$

which is a function of $\tau$. When $\tau$ is unconstrained,

$$
\tau(1-\tau) \leq\left(\frac{\tau+(1-\tau)}{2}\right)^{2}=\frac{1}{4}
$$

with maximum at $\tau_{\mathrm{m}}=1 / 2$. However, since $\tau$ is constrained between $[F, H]$, there are three possible scenarios: $\tau_{\mathrm{m}}=1 / 2$ when $F \leq 1 / 2 \leq H, \tau_{\mathrm{m}}=H$ when $F \leq H<1 / 2$, and $\tau_{\mathrm{m}}=F$ when $1 / 2 \leq F<H$. They lead to the corresponding maximum areas in Proposition 1 .

\section{A Correct Formula for Non-parametric Sensitivity}

Given the results in the previous section, neither Pollack and Norman's (1964) $A^{\prime}$ nor Smith's (1995) $A^{\prime \prime}$ provides an accurate measure of the common interpretation of non- parametric sensitivity index as the average of the maximum area and minimum area proper ROC curves constrained by the experimental data $(F, H)$. We provide here the correct formula (which we denote $A$ ) for the average of $A_{\max }(3)$ and $A_{\min }=(1+H-F) / 2$ :

$$
A=\left\{\begin{array}{llc}
\frac{3}{4}+\frac{H-F}{4}-F(1-H) & \text { if } \quad F \leq 0.5 \leq H \\
\frac{3}{4}+\frac{H-F}{4}-\frac{F}{4 H} & \text { if } \quad F \leq H<0.5 ; \\
\frac{3}{4}+\frac{H-F}{4}-\frac{1-H}{4(1-F)} & \text { if } & 0.5<F \leq H .
\end{array}\right.
$$

$A$ is the correct formula for determining the average of the minimum-area proper ROC curve and the maximum-area proper ROC curve. However, this measure ignores the fact that the minimum-area and maximum-area proper ROC curves might differ considerably for some constraining points, whereas they might be very similar for others. Consequently, one might be reasonably confident about $A$ in regions where the maximum and minimum areas are nearly the same, and be more hesitant about using $A$ for data in regions where there is a large difference between the minimum-area and maximum-area proper ROC curves.

Figure 3 examines this issue, by plotting the difference between the minimum-area and maximum-area proper ROC curves that can pass through each point. The figure shows that the smallest differences occur along the positive and negative diagonals of the ROC space, especially for points close to $(0,1)$ and to $(0.5,0.5)$. The points where there is the greatest difference between minimum and maximum-area proper ROC curves are near the lines $H=0$ and $F=1$. Thus, data observed near these boundaries of the ROC space may result from proper ROC curves with a large range of underlying areas. Consequently, care should be taken when using the $A$ measure to interpret data observed in these latter regions. Note that these are also the regions where $d^{\prime}$ would change most rapidly with respect to a change in $H$ or $F$, and the regions where slightly different assumptions about distributions can have the largest impact.

One way to examine $A$ and related area-based measures of sensitivity is to examine the "iso-sensitivity" curve, i.e., the combinations of $F$ and $H$ that will produce a given value of $A$. The topography of $A$ in the ROC space can be mapped by drawing isopleths for its different constant values. Figure 4 shows these topographic maps for $A^{\prime}, A^{\prime \prime}$, and $A$, in equal increments of sensitivity values ( 0.05 per increment). It can be noticed that $A^{\prime \prime}$ has a sharp discontinuous 


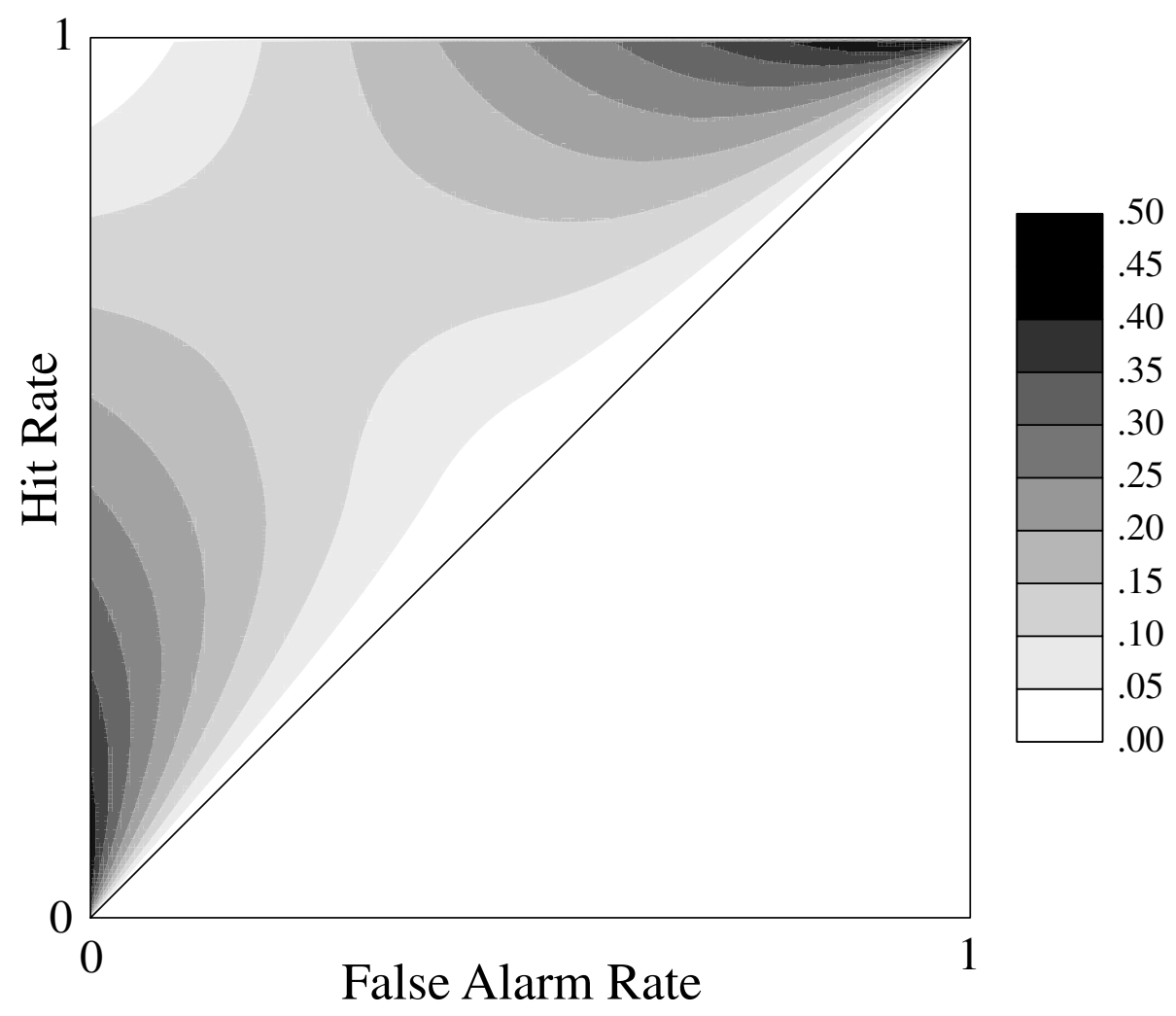

FIGURE 3.

Difference between the minimum-area and maximum-area proper ROC curves through a point in ROC space. Lighter regions indicate smaller differences (in 0.05 increments).

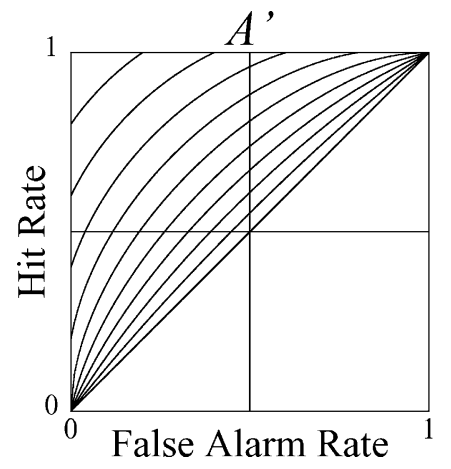

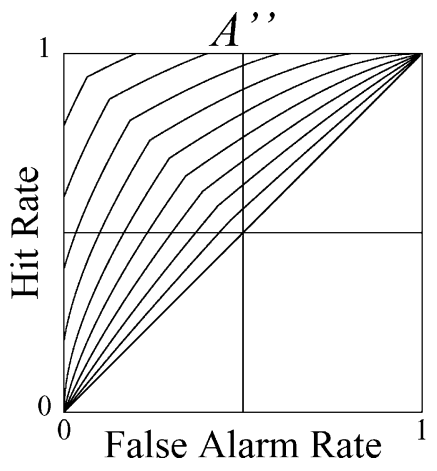

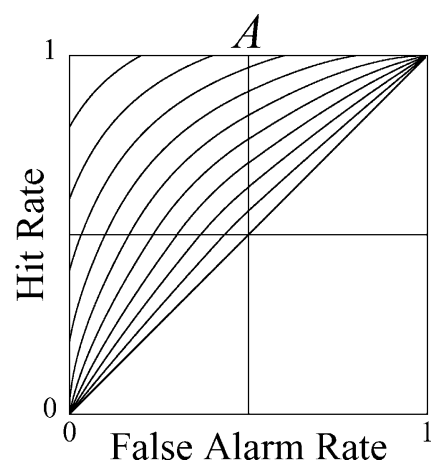

FIGURE 4.

Constant-sensitivity isopleths for $A^{\prime}, A^{\prime \prime}$, and $A$. Each curve represents combinations of $F$ and $H$ that produce equal values of sensitivity, in increments of $0.05\left(A^{\prime}, A^{\prime \prime}\right.$, or $A$ in the left, middle, and right panels, respectively).

point along the negative diagonal $H+F=1$. Each constant- $A$ isopleth lies slightly above the constant- $A^{\prime}$ isopleth of the same corresponding value.

Note that the actual differences between these formulae $\left(A^{\prime}, A^{\prime \prime}\right.$ and $A$ ) are relatively small. However, the differences are greatest in the upper-left quadrant of the ROC space, especially along or near the negative diagonal, a region where empirical data most commonly fall under. 


\section{An Associated Measure of Decision Bias: $b$}

As shown in the next section, the slope of any proper ROC curve is related to the likelihood ratio of the underlying distributions (for signal and for noise), and as such, it can be used as an index of decision bias. Consequently, we propose that the slope of the constant- $A$ curve through a point can be used as an index of decision bias or criterion. This slope $(b)$ can be found by the following formula:

$$
b= \begin{cases}\frac{5-4 H}{1+4 F} & \text { if } \quad F \leq 0.5 \leq H \\ \frac{H^{2}+H}{H^{2}+F} & \text { if } \quad F \leq H<0.5 \\ \frac{(1-F)^{2}+(1-H)}{(1-F)^{2}+(1-F)} & \text { if } \quad 0.5<F \leq H .\end{cases}
$$

The isopleths of $b$ are shown in Figure 5.

\section{Slope Monotonicity and Likelihood-ratio Based Criterion}

The above derivation of the A measure (in fact of all area-based measures) relies on the assumption that the corresponding ROC curves are "proper" (see Footnote 1). That an ROC curve has non-increasing slope is not always guaranteed, e.g., when the signal and the noise distributions are normal distributions with unequal variance. However, slope-monotonicity will be shown to hold when decision is based on a cutoff of the likelihood-ratio value.

According to the traditional signal detection framework, an ROC curve $u_{\mathrm{c}} \mapsto\left(F\left(u_{\mathrm{c}}\right), H\left(u_{\mathrm{c}}\right)\right)$ is parameterized by the cutoff criterion value $u_{\mathrm{c}}$ along the measurement (sensory encoding) axis on

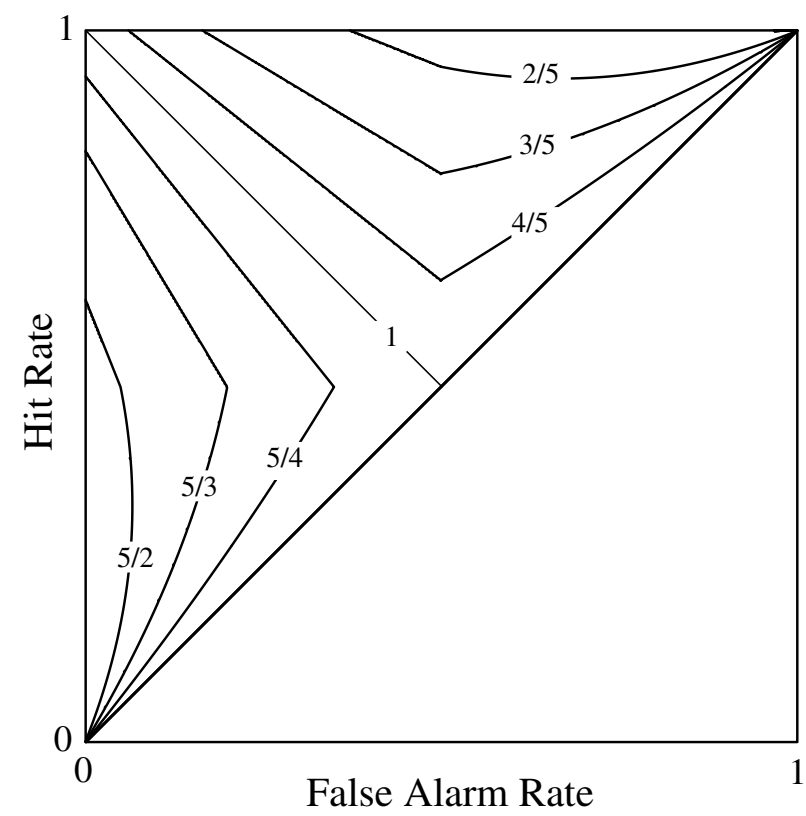

FIGURE 5 .

Isopleths for constant values of $b$. The values of $b$ produced by combinations of $F$ and $H$ are symmetric around $H=1-F$ (for which $b=1$ ). For a given value of $b$, its corresponding symmetric isopleth has a value of $1 / b$. 
which a decision is based. The signal and noise distributions may be generated by systematically varying the signal strength while recording the subject response. Given the underlying signal distribution $f_{\mathrm{s}}(u)$ and noise distribution $f_{\mathrm{n}}(u)$ of measurement (encoded) value $u$, a criterion-based response strategy will give rise to

$$
H\left(u_{\mathrm{c}}\right)=\int_{u_{\mathrm{c}}}^{\infty} f_{\mathrm{s}}(u) d u, \quad F\left(u_{\mathrm{c}}\right)=\int_{u_{c}}^{\infty} f_{\mathrm{n}}(u) d u .
$$

The slope of the ROC curve at criterion $u_{\mathrm{c}}$ is

$$
\left.\frac{d H}{d F}\right|_{F=F\left(u_{\mathrm{c}}\right), H=H\left(u_{\mathrm{c}}\right)}=\frac{H^{\prime}\left(u_{\mathrm{c}}\right)}{F^{\prime}\left(u_{\mathrm{c}}\right)}=\frac{f_{\mathrm{s}}\left(u_{\mathrm{c}}\right)}{f_{\mathrm{n}}\left(u_{\mathrm{c}}\right)} \equiv l\left(u_{\mathrm{c}}\right) .
$$

So the requirement of a proper ROC curve, i.e., one that has non-increasing slope as the cutoff criterion $u_{\mathrm{c}}$ decreases, is equivalent to the monotonicity of the function $l(u)$.

It is well known (see Green \& Swets, 1964) that a monotone transformation of the measurement (sensory encoding) axis does not change the shape of the ROC curve (since it is just a re-parameterization of the curve). Complications arise when the likelihood function $l(u)$ is a non-monotonic (still assumed to be continuous and differentiable) function of the sensory encoding variable $u$. In this case, however, one can define the decision criterion by a cutoff value in likelihood ratio $l_{\mathrm{c}}$, with the corresponding hit rate $(H)$ and false-alarm rate $(F)$ given by

$$
H\left(l_{\mathrm{c}}\right)=\int_{\left\{u: l(u)>l_{\mathrm{c}}\right\}} f_{\mathrm{s}}(u) d u, \quad F\left(l_{\mathrm{c}}\right)=\int_{\left\{u: l(u)>l_{c}\right\}} f_{\mathrm{n}}(u) d u .
$$

It can be shown that in this case, we may still have:

Proposition 2. The slope of a likelihood-ratio based ROC curve $l_{\mathrm{c}} \mapsto\left(F\left(l_{\mathrm{c}}\right), H\left(l_{\mathrm{c}}\right)\right)$ parameterized by likelihood ratio is monotonically decreasing going from $(0,0)$ to $(1,1)$.

Proof. We only need to show that, for an ROC curve generated by a decision mechanism using the likelihood-ratio criterion, its slope at criterion $l_{\mathrm{c}}$ exactly equals $l_{\mathrm{c}}$

$$
\frac{d H\left(l_{\mathrm{c}}\right)}{d F\left(l_{\mathrm{c}}\right)}=l_{\mathrm{c}} \text {. }
$$

It follows that the slope (left-side of the above) must be decreasing as the criterion decreases.

To prove slope-monotonicity of ROC curves parameterized by $l_{\mathrm{c}}$, we calculate $H\left(l_{\mathrm{c}}+\delta l\right)-$ $H\left(l_{\mathrm{c}}\right) \equiv \delta H\left(l_{\mathrm{c}}\right)$ explicitly as

$$
\begin{aligned}
\delta H\left(l_{\mathrm{c}}\right) & =\int_{\left\{u: l(u)>l_{\mathrm{c}}+\delta l\right\}} f_{\mathrm{s}}(u) d u-\int_{\left\{u: l(u)>l_{\mathrm{c}}\right\}} f_{\mathrm{s}}(u) d u \\
& =-\int_{\left\{u: l_{\mathrm{c}}<l(u)<l_{\mathrm{c}}+\delta l\right\}} f_{\mathrm{s}}(u) d u \simeq-\sum_{\left\{i: l\left(u_{i}\right)=l_{\mathrm{c}}\right\}} f_{\mathrm{s}}\left(u_{i}\right) \delta u_{i},
\end{aligned}
$$

where $\delta u_{i}=\left(\left.\frac{d l}{d u}\right|_{u=u_{i}}\right)^{-1} \delta l$, and the summation is over an index set $I$ such that $l\left(u_{i}\right)=l_{\mathrm{c}}, i \in I$. In other words, the $u_{i}$ 's are the set of all pre-image points corresponding to the given $l=l_{\mathrm{c}}$ value, of the non-monotonic map $u \mapsto l(u)$. Similarly,

$$
\delta F\left(l_{\mathrm{c}}\right) \simeq-\sum_{\left\{i: l\left(u_{i}\right)=l_{\mathrm{c}}\right\}} f_{\mathrm{n}}\left(u_{i}\right) \delta u_{i}
$$


Now, because

$$
\frac{f_{\mathrm{s}}\left(u_{i}\right)}{f_{n}\left(u_{i}\right)}=l\left(u_{i}\right)=l_{\mathrm{c}}
$$

is a constant for all $i \in I$, from an elementary theorem on ratios, which says that if $a_{i} / b_{i}=$ $c$ for $i \in I$ (where $c$ is a constant and $I$ in an index set), then $\left(\sum_{i \in I} a_{i}\right) /\left(\sum_{i \in I} b_{i}\right)=c$,

$$
\frac{\delta H\left(l_{\mathrm{c}}\right)}{\delta F\left(l_{\mathrm{c}}\right)}=\frac{\sum_{\left\{i: l\left(u_{i}\right)=l_{\mathrm{c}}\right\}} f_{\mathrm{s}}\left(u_{i}\right) \delta u_{i}}{\sum_{\left\{i: l\left(u_{i}\right)=l_{\mathrm{c}}\right\}} f_{\mathrm{n}}\left(u_{i}\right) \delta u_{i}}=l_{\mathrm{c}} .
$$

Taking limit $\delta l \rightarrow 0$ concludes the proof.

The above conclusion also holds for multidimensional observations, i.e., when evidence consists of a multi-dimensional observation vector $\mathbf{u}$, with density of likelihood function $f_{\mathrm{s}}(\mathbf{u})$, $f_{\mathrm{n}}(\mathbf{u})$ (e.g., Ashby \& Townsend, 1986). Using the likelihood ratio $l(\mathbf{u})=\frac{f_{\mathrm{s}}(\mathbf{u})}{f_{\mathrm{n}}(\mathbf{u})}$ as the decision axis and $l_{\mathrm{c}}$ as the cutoff criterion, the hit and false-alarm rates are

$$
H\left(l_{\mathrm{c}}\right)=\int_{\left\{\mathbf{u}: l(\mathbf{u}) \geq l_{\mathrm{c}}\right\}} f_{\mathrm{s}}(\mathbf{u}) d \mathbf{u}, \quad F\left(l_{\mathrm{c}}\right)=\int_{\left\{\mathbf{u}: l(\mathbf{u}) \geq l_{\mathrm{c}}\right\}} f_{\mathrm{n}}(\mathbf{u}) d \mathbf{u} .
$$

It can be analogously shown that

$$
\frac{d H\left(l_{\mathrm{c}}(\mathbf{u})\right)}{d F\left(l_{\mathrm{c}}(\mathbf{u})\right)}=l_{\mathrm{c}}(\mathbf{u}) .
$$

Therefore, whether the likelihood ratio is monotonically or non-monotonically related to the sensory dimension and whether the likelihood ratio is based on uni- or multi-dimensional signals, the slope of the ROC curve is always equal to the likelihood-ratio value regardless of how it is parameterized. Decisions based on the likelihood ratio are optimal among all criterion-based decision schemes. The Neyman-Pearson Lemma guarantees that such an ROC curve (which is necessarily proper) lies above ROC curves (which may not be proper) using any other criterion. By assuming that ROC curves are proper in defining $A_{\max }$, we assume that subjects base their decisions on the likelihood ratio rather than on the sensory encoding axis. ${ }^{2}$

This likelihood-ratio based analysis is seen (Balakrishnan, 1998; 1999) as an alternative to the traditional stimulus-based paradigm. Using confidence rating as measurements, Balakrishnan $(1998,1999)$ showed that the likelihood ratio is a monotonic (though not identical) function of the confidence rating. Under manipulation of prior odds, the criterion value of confidence rating is unchanged in a classification task where the decision criterion is uniformly unbiased. Under the likelihood-ratio parameterization, the signal distribution $f_{s}(l)=-d H / d l$ and the noise distribution $f_{\mathrm{n}}(l)=-d F / d l$ can be shown to satisfy (i) $E_{\mathrm{n}}\{l\}=\int l f_{\mathrm{n}}(l) d l=1$ and (ii) $E_{\mathrm{s}}\{l\}=\int l f_{\mathrm{s}}(l) d l \geq 1$.

\section{Conclusion and Discussion}

We provided a correct formula for the maximal area of proper ROC curves passing through a data point and, through that, a correct formula for the non-parametric estimate of sensitivity as

\footnotetext{
${ }^{2}$ Note that likelihood-ratio based ROC curves may not be continuous when the index set $I$ as introduced in the proof above is not measure zero; this occurs when $I$ contains a dense subset $U$ of the real line (the measurement axis $u$ ) whose elements all have identical likelihood-ratio value. In this case, the line connecting the two discontinuous ROC points is interpreted as the subject's varying criteria based on $u$ among all those points $u \in U$ with a fixed likelihood-ratio value $l(u)$.
} 
defined by the average of maximal and minimum ROC areas. We also demonstrated slope-monotonicity for a likelihood-ratio based ROC curve despite a non-monotonic transformation from the measurement axis (encoding values). The relationship of the ROC slope to the likelihood ratio is a fundamental relation in ROC analysis, as it is invariant with respect to any continuous reparameterization of the stimulus, including non-monotonic mapping of uni-dimensional and multi-dimensional signals in general.

A cautionary note about ROC area measures in general is warranted. An ROC curve is, in essence, two cumulative probability distributions (of signal and of noise respectively), regardless of which particular parametric families (normal, exponential, gamma, etc.) they come from or whether they are parametric at all. Hence, measures of sensitivity based on the area under an ROC curve does not rely on any distributional assumptions, and can be used as a numerical index to characterize the "distance" or "separation" between two probability distributions-for instance, in analyzing single neuron responses, Newsome, Britten, Movshon, and Shadlen (1989) applied the area measure to describe a neuron's discriminability to two stimuli, whereas Zhang, Riehle, and Requin (1997) characterized a neuron's sensorimotor locus based on area measures derived from stimulus- and response-conditioned analyses. However, the distribution-free nature of the area measure does not imply that the maximum- and/or minimum-area ROC curves constrained to pass through a given point are immune from any distributional interpretations, as pointed out by Macmillan and Creelman (1996). There is also the broader question of whether an ROC curve truly reflects isosensitivity manipulations, since manipulation of bias/prior odds may not have affected the likelihood-ratio at which a decision is rendered (Balakrishnan 1998, 1999). While care must be taken in invoking the popular measure (namely $A^{\prime}$ ), this note provides a correct formula (namely $A$ ) for its calculation, and has thus settled once and forever the issue of finding the "average of the areas subtended by the upper, and by the lower bounds" of proper ROC curves passing through a given data point as first raised by Pollack and Norman (1964, p. 126).

References

Ashby, F.G., \& Townsend, J.T. (1986). Varieties of perceptual independence. Psychological Review, 93, 154-179

Balakrishnan, J.D. (1998). Some more sensitive measures of sensitivity and response bias. Psychological Methods, 3 , $68-90$.

Balakrishnan, J.D. (1999). Decision processes in discrimination: fundamental misrepresentations of signal detection theory. Journal of Experimental Psychology: Human Perception and Performance, 25, 1189-1206.

Donaldson, W., \& Good, C. (1996). A'r: An estimate of area under isosensitivity curves. Behavioral Methods, Instruments, and Computers, 28, 590-597.

Green, D.M., \& Swets, J.A. (1964). Signal Detection Theory and Psychophysics, New York: John Wiley \& Sons.

Macmillan, N.A., \& Creelman, C.D. (1996). Triangles in ROC space: History and theory of "non-parametric" measures of sensitivity and response bias. Psychonomic Bulletin and Review, 3, 164-170.

Newsome, W.T., Britten, K.H., Movshon, J.A., \& Shadlen, M. (1989) Single neurons and the perception of visual motion. In D.M-K. Lam \& C.D. Gilbert (Eds.) Proceedings of the Retina Research Foundation Symposia, Vol. 2, Neural Mechanisms of Visual Perception, (pp. 171-198). Woodlands, TX: Portfolio Publishing.

Peterson, W.W., Birdsall, T.G., \& Fox, W.C. (1954). The theory of signal detectability. Transactions of the IRE Professional Group on Information Theory, PGIT-4, 171-212.

Pollack, I., \& Hsieh, R. (1969). Sampling variability of the area under ROC curve and $d_{e}^{\prime}$. Psychological Bulletin, 1 , $161-173$.

Pollack, L., \& Norman, D.A. (1964). Non-parametric analysis of recognition experiments. Psychonomic Science 1, 125126.

Smith, W.D. (1995). Clarification of sensitivity measure A'. Journal of Mathematical Psychology 39, 82-89.

Swets, J.A., Tanner, W.P., \& Birdsall, T.G. (1964). Decision process in perception. Psychological Review 68, 301-340.

Tanner, W.P., Jr., \& Swets, J.A. (1954). A decision-making theory of visual detection. Psychological Review 61, 401-409.

Zhang, J., Riehle, A., \& Requin, J. (1997). Locus of a neural process in stimulus-response association tasks. Journal of Mathematical Psychology 41, 219-236.

Manuscript received 18 AUG 2003

Final version received 22 JAN 2004 